\title{
Taking the quantum eraser to the abstract world
}

Nape, I., Ndagano, B., Forbes, A.

I. Nape, B. Ndagano, A. Forbes, "Taking the quantum eraser to the abstract world," Proc. SPIE 10409, Quantum Communications and Quantum Imaging XV, 1040906 (30 August 2017); doi: 10.1117/12.2274173

EDent: SPIE Optical Engineering + Applications, 2017, San Diego, California, United States 


\title{
Taking the quantum eraser to the abstract world
}

\author{
Nape I. ${ }^{1}$, Ndagano B. ${ }^{1}$, and Forbes A. ${ }^{1}$ \\ ${ }^{1}$ University of the Witwatersrand, 1 Jan Smuts Avenue, Johannesburg, South Africa
}

\begin{abstract}
Youngs double slit experiment is one of the most celebrated achievements in quantum and classical optics; it provides experimental proof of the wave-particle duality of light. When the paths of the double slit are marked with orthogonal polarizations, the path information is revealed and no interference pattern is observed. However, the path information can be erased with a complimentary analysis of the polarization. Here we use hybrid entanglement between photons carrying orbital angular momentum and polarization to show that, just as in Young's experiment, the paths (OAM) marked with polarization do not lead to interference. However, when introducing the eraser (polarizer) which projects the polarization of one of the entangled photons onto a complementary polarization basis, the OAM (paths) are allowed to interfere, leading to the formation of azimuthal fringes whose frequency is proportional to the OAM content carried by the photon.
\end{abstract}

Keywords: quantum eraser, intereference, orbital angular momentum, hybrid entaglement

\section{INTRODUCTION}

The wave-like behavior of photons and matter waves is an interesting feature that has been revealed through a myriad of double slit and path interferometric experiments. ${ }^{1,2,3,4,5}$ Interestingly, these quantum systems also exhibit a contrasting property, a particle like behavior, which cannot be observed simultaneously according to the complementarity principle. ${ }^{6}$ This can be verified through the fringe visibility and path distinguishably of photons in the double slit experiment. ${ }^{7,8,9,10}$ The former indicative of a wave-like and the latter a particle like behavior. Exploiting this feature has enabled for quantum eraser experiments to be performed, where path information (particle-like) can be erased from a quantum system. ${ }^{11,12}$

Significantly, most variations of the quantum eraser have exploited double slits ${ }^{13,14,15}$ and beam splitters. ${ }^{16,17,18}$ Entangled photons have also been exploited. For example in ref. ${ }^{13}$ the particle or wave-like behavior of one photon is heralded by another, ultimately enabling for the obtainment and removal of the path information in the system. Moreover, delayed choice versions have been reported ${ }^{18,19,20}$ wherein non-locality plays a vital role. However most reports of the quantum eraser have limited the experiment to physical path interference although in quantum mechanics a path can be any abstract variable.

In this paper, we show that quantum eraser can be extended to the abstract paths of orbital angular momentum (OAM). ${ }^{21,22,23,24}$ We use polarization as our OAM equivalent path marker through polarization-OAM hybrid entanglement. We engineer the hybrid entanglement from spontaneous parametric down conversion and perform a orbit to spin conversion using geometric phase control. We show that through the polarization control of one of the entangled photons, the OAM (paths) marked with polarization do not lead to OAM (path) interference (introducing OAM information). We erase the OAM information with a complementary polarization projection of the twin photon. We determine whether the OAM is marked or erased by scanning the azimuthal distribution of the photons and measuring the fringe visibility. We obtained low $(\mathcal{V} \approx 0.04)$ and high $(\mathcal{V} \approx 0.92)$ visibilities showing that we can determine and erase the OAM information in the system.

\section{THEORY}

\subsection{Traditional quantum eraser}

The entanglement based version of the quantum eraser can be illustrated by considering the following hybrid path-polarization entangled state

Further author information: E-mail: isaacnape@gmail.com 

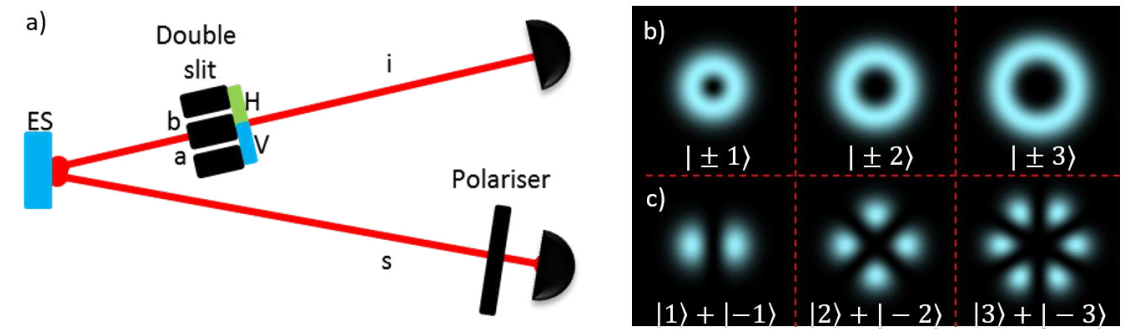

Figure 1. (a) A concept schematic of the quantum eraser with polarized entangled photons. The entangled photons are spatially separated into the signal (s) and idler (i) arms. The double slit is marked with linear horizontal(H) and vertical (V) polarizer is used to judiciously obtain and erase the path information in the system. (b) An illustration of the intensity distribution of OAM eigenmodes and their (c) superposition states.

$$
|\Psi\rangle_{s, i}=\frac{1}{\sqrt{2}}\left(|H\rangle_{s}|a\rangle_{i}+|V\rangle_{s}|b\rangle_{i}\right)
$$

Here $|H\rangle_{s}$ and $|V\rangle_{s}$ represent the horizontal and vertical linear polarization states of the signal ( $s$ ) photon that mark paths $a$ and $b$ of the idler $(i)$ photon with eigenstates $|a\rangle_{i}$ and $|b\rangle_{i}$, respectively. The system in Equation. (1) can be generated by allowing the signal photon to interact with a physical double slit just as in ref. ${ }^{13}$ The eraser procedure can be carried out as follows: first particle information is introduced into the system by projecting photon $s$ onto the state $|H\rangle_{s}\left(|V\rangle_{s}\right)$ resulting in the obtainment of path information in the system indicating that the photon traverses path $|a\rangle_{i}\left(|b\rangle_{i}\right)$ (particle-like behavior). Next the path information can be erased by projecting the signal photon onto a superposition state, i.e., $| \pm\rangle=\frac{1}{\sqrt{2}}(|H\rangle \pm|V\rangle)$ using a polarizer, resulting in

$$
|\Psi\rangle_{s, i} \rightarrow \frac{1}{\sqrt{2}}| \pm\rangle_{s}\left(|a\rangle_{i} \pm|b\rangle_{i}\right)
$$

where the idler photon collapses onto a superposition even though the paths were initially marked, consequently allowing them to interfere (a wavelike property). Here, the polarizer acts as an eraser; it removes the path information from the system. Remarkably, the particle and wave like nature are deduced form the interference fringe visibility $\mathcal{V}$. When the paths are marked $\mathcal{V}=1$ and once erased $\mathcal{V}=0$. This is a characteristic of the complementarity principle which can be quantitatively written as

$$
\mathcal{V}^{2}+\mathcal{D}^{2} \leq 1
$$

where $\mathcal{D}$ is the path information. The special cases $(\mathcal{D}=1, \mathcal{V}=0)$ and $(\mathcal{D}=0, \mathcal{V}=1)$ correspond to maximal path information obtainment and maximal eraser, respectively.

\subsection{Abstracting path to orbital angular momentum}

The path eigenstates in Eq. (1) can be treated as a two level (qubit) system on the projective Hilbert space. Since the Hilbert space is not restricted to the path DoF, one can replace the notion of path with an alternative DoF, i.e., the orbital angular momentum (OAM) of photons. The OAM eigenstates can be represented by the state $| \pm \ell\rangle$ corresponding to a characteristic azimuthal phase profile $e^{i \ell \phi}$ where each photon carries an angular momentum of $\pm \ell \hbar$ with the integer $\ell$ representing the topological charge. ${ }^{23}$

A qubit space can be constructed for OAM modes with opposite topological charges, i.e, $\mathcal{H}_{2}=\operatorname{span}\{|\ell\rangle,|-\ell\rangle\}$. Analogously, each OAM eigenmodes can be treated as an abstract path. The OAM eigenmodes, $|\ell\rangle$ and $|-\ell\rangle$ have a uniform intensity distribution (see Fig. 1 (b)) while the azimuthal phase profiles are opposite in rotation. The state $|\ell\rangle$ is right handed while the state $|-\ell\rangle$ is left handed. Moreover, superposition states, $|\ell\rangle \pm|-\ell\rangle$, give rise to azimuthal spatial fringes with a frequency of $2 \ell$. This is illustrated in Fig. 1 (c) for $|\ell|=1,2,3$ where spatial fringes are observed in the azimuthal direction. Analogous to the appearance of lateral interference fringes from 


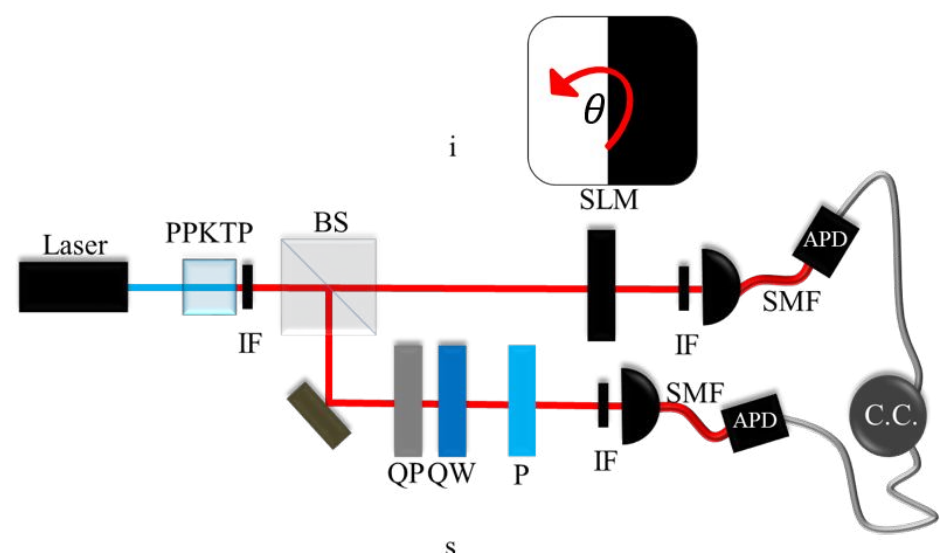

Figure 2. (a)Experimental set-up. A periodically-poled-potassium-titanyl-phosphate (PPKTP) crystal was pumped with a $405 \mathrm{~nm}$ laser in a collinear set-up; the entangled photons were produced on axis with the pump. The entangled photons were split into the signal (s) and idler (i) arm with a 50/50 beam-splitter (BS). We engineered an orbit to spin conversion in arm s with a $q$-plate $(\mathrm{QP})(q=0.5)$. Using a quarter wave-plate $(\mathrm{QW})$ and with a polarizer $(\mathrm{P})$ we marked and erased the OAM of the idler photon. The down-converted photons were filtered with 10nm bandwidth interference filter (IF). Single mode fibers (SMF) where coupled to avalanche photo-diodes (APD) and counted the photons in coincidence with a coincidence counter (C.C).

path interference, we can use the azimuthal fringe visibility to determine whether there is OAM information or whether it has been erased from the system. Given the above motivation, we can rewrite Eq. (1) as

$$
|\psi\rangle_{s, i}=\frac{1}{\sqrt{2}}\left(|H\rangle_{s}|\ell\rangle_{i}+|V\rangle_{s}|-\ell\rangle_{i}\right),
$$

where the path eigenstates are replaced by abstract paths of OAM.

\section{EXPERIMENT}

In Fig. 2.(a), we present the experimental set-up for our quantum eraser. We engineered hybrid entanglement from spontaneous parametric down conversion (SPDC) and geometric phase control. A PPKTP nonlinear crystal, cut for type 1 phase matching, was pumped with a $405 \mathrm{~nm}$ Coherent Cube laser with a Gaussian profile and produced $810 \mathrm{~nm}$ entangled photon pairs given by the following state

$$
|\Psi\rangle_{S P D C}=\sum_{\ell} c_{|\ell|}|\ell\rangle_{s}|-\ell\rangle_{i}|H\rangle_{s}|H\rangle_{i},
$$

where $\left.c_{\mid} \ell\right|^{2}$ is the probability of finding the signal and idler photon in the state $| \pm \ell\rangle$, conserving the orbital angular monument of the pump photon. The entangled photons were separated with a 50/50 BS. We performed a orbit-to-spin conversion of the idler photon using a $q$-plate, ${ }^{25,26,27,28}$ an optical element that performs spinorbit coupling. Noting that $|H\rangle_{s}=\left(|R\rangle_{s}+|L\rangle_{s}\right) / \sqrt{2}$ and subsequently applying the transformation of the $q$-plate on the signal photon, Eq. (5) becomes $^{29}$

$$
|\Psi\rangle_{s, i}=\frac{1}{\sqrt{2}}\left(|R\rangle_{s}|\ell\rangle_{i}+|L\rangle_{s}|-\ell\rangle_{i}\right)
$$

where $\ell=1$ since $q=0.4$ OAM subspaces. Next we rotated from circular to linear polarization using a QW wave plate, inserted after the $q$-plate and oriented at $45^{\circ}$ resulting in the state 


$$
|\psi\rangle=\frac{1}{\sqrt{2}}\left(|H\rangle_{s}|\ell\rangle_{i}+i|V\rangle_{s}|-\ell\rangle_{i}\right)
$$

We point out that the OAM "path" was marked by orthogonal linear polarization states. A polarizer was used to distinguish and eraser the OAM information in the system. The target state is given by

$$
|\alpha\rangle_{s}=\cos (\alpha)|H\rangle_{s}+\sin (\alpha)|V\rangle_{s}
$$

Here $\alpha$ defines the angle of the polarizer. The azimuthal distribution of the photons can be analyzed by encoding a binary phase step $(0$ and $\pi)$ corresponding to the state $|\theta\rangle_{B}=\left(|\ell\rangle_{B}+e^{i 2 \theta}|-\ell\rangle_{B}\right)$ with an orientation proportional to the relative phase, $(\theta)$, between the orthogonal $|\ell\rangle$ and $|-\ell\rangle$ OAM states. ${ }^{30,31,32,33}$ The phase step was encoded onto the SLM, in the signal arm, and served as a pattern sensitive scanner. ${ }^{34,35}$ A Holoeye PLUTO phase only SLM was used to encode the binary phase mask using the digitally controlled display enabling us to scan the azimuthal spatial distribution of the photon in the idler arm. A gating time of $25 \mathrm{~ns}$ was used as a window within which coincidence events between the idler and signal were collected from the APDs.

The theoretical probability of detection is given by $^{29}$

$$
\begin{aligned}
P(\alpha, \theta) & \propto \mid\left\langle\left.\left.\theta\right|_{B}\left\langle\left.\alpha\right|_{A} \mid \psi\right\rangle_{A B}\right|^{2}\right. \\
& =\frac{1}{2}(1+\sin (2 \alpha) \cos (2 \theta+\delta)) .
\end{aligned}
$$

where $\delta=\frac{\pi}{2}$. The visibility of the interference fringes is proportional to $|\sin (2 \alpha)|$ by Eq. (9). We varied the angle of the polarizer from 0 to $45^{\circ}$ subsequently scanning of the azimuthal photon distribution to determine the fringe visibility.

\section{RESULTS}
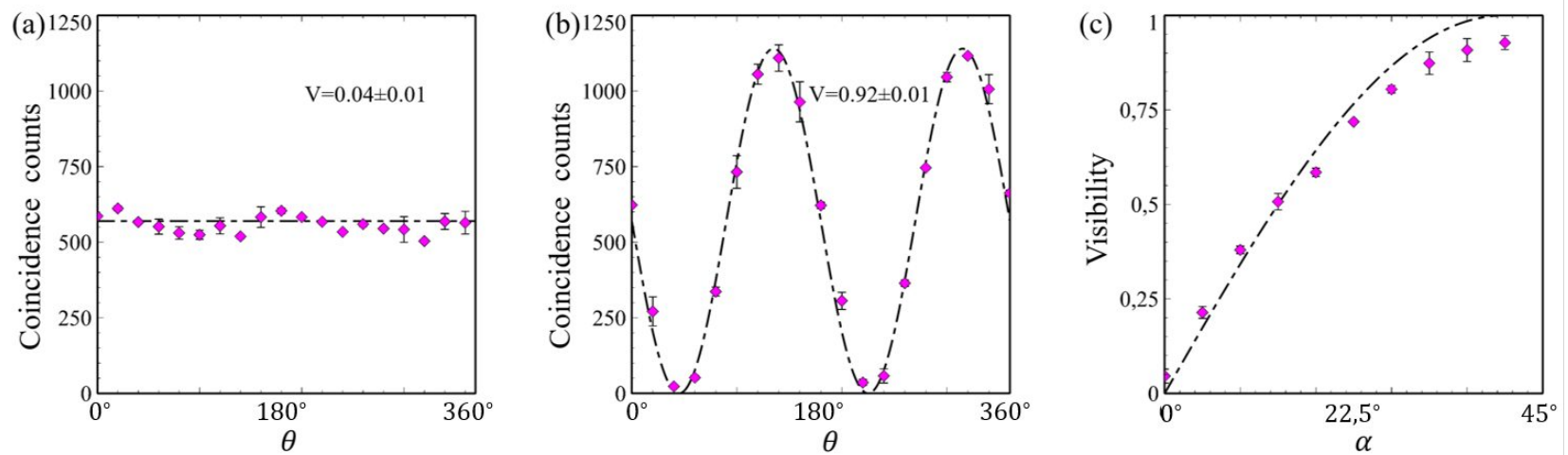

Figure 3. Experimental coincidence counts and visibility measurements. The purple points represent the experimental results and dashed lines represent the theoretical plot. (a) and (b) are coincidence counts when $\alpha=0$ (OAM information is obtained) and $\alpha=45^{\circ}$ (erased) with low and high visibilities, receptively The visibility measurements with a variation of the polarizer are presented in (c) showing minimal visibility when the OAM is marked and maximal visibility when it is erased.

We present the experimental results. The OAM information of the idler photon was obtained by projecting the polarization of the signal photon onto the state $|H\rangle$ corresponding to $\alpha=0^{\circ}$. The angular mask was encoded on the SLM and rotated while the signal and idler photons where detected in coincidence. The results are presented in Fig. 3(a) where there are minimal fringes observed. The small oscillations are due to imperfections 
in the polarization filtering of the signal photon. Our results are in accordance with Eq. (9) which predicts $P(\theta)=$ constant for $\alpha=0$ indicating that there is OAM information in the system. Furthermore, the measured visibility of the spatial fringes is $0.04 \pm 0.01$ indicating that there is no OAM interference, analogous to identifying the path of a photon in the double slit experiment.

Next, we erased the OAM information with a judicious choice of the polarizer angle by setting it to $\alpha=-45^{\circ}$ thus selecting the $|-\rangle$ polarization state. The coincidence counts obtained from the azimuthal scanning are presented in Fig. 3 (b) where interference fringes with a visibility of $0.92 \pm 0.01$ were measured indicating that the OAM information is erased. The oscillating counts is consistent with Eq. (9).

Lastly, we measured the visibility against $\alpha$ in the range $\alpha=\left[0^{\circ}, 45^{\circ}\right]$ increments of $5^{\circ}$. The results are presented in Fig. 3 (c). The interference fringes are minimal at $\alpha=0$ and maximal when $\alpha=45^{\circ}$. Furthermore, visibility of the fringes is proportional to $|\sin (2 \alpha)|$, as predicted by the theory (Eq. (9)).This indicates that a reduction in the OAM information increases the visibility indicating a complementarity between the OAM information and the azimuthal spatial fringe visibility.

\section{DISCUSSION AND CONCLUSION}

We have shown that the OAM of a photon can be erased through polarization control in a hybrid entangled system and our results are in agreement with the theory. Our results indicate that marking the OAM of a photon with polarization of its entangled twin does not lead to azimuthal fringes while a complimentary measurement of the polarization lead to highly visible interference fringes (wave-like) and in other words erases the OAM information.

Here the role of hybrid entanglement has a vital role which allowed us to abstract the concept of path to OAM and exploit entanglement between polarization and OAM reminiscent to path polarization hybrid entanglement. We have also reported an extension of these results, where we performed the eraser procedure in a delayed measure scheme. ${ }^{29}$ Furthermore, this new way of looking at the quantum eraser has proven to be invaluable for quantum communication; we have recently shown that our scheme capable of revealing the amount of disturbance present in a quantum channel ${ }^{36}$ relevant for the quantum communication with spatial modes of light. ${ }^{37,38,39} \mathrm{We}$ point out that any other DoF could have been used and chose orbital angular momentum due to the recent interest in applications for quantum information and communication.

In conclusion we have demonstrated the advantage of using OAM as an abstract path. This does not require any complicated interferometers and enabled us to perform the eraser procedure with ease. We showed that the fringe visibility and OAM information acquisition are complimentary.

\section{ACKNOWLEDGMENTS}

The authors express their gratitude to Lorenzo Marrucci for providing $q$-plates. B.N. acknowledges financial support from the National Research Foundation of South Africa and I. N. from the Department of Science and Technology (South Africa).

\section{REFERENCES}

[1] Rauch, H., Treimer, W., and Bonse, U., "Test of a single crystal neutron interferometer," Physics Letters A 47(5), 369-371 (1974).

[2] Grangier, P., Roger, G., and Aspect, A., "Experimental evidence for a photon anticorrelation effect on a beam splitter: a new light on single-photon interferences," EPL (Europhysics Letters) 1(4), 173 (1986).

[3] Zeilinger, A., Gähler, R., Shull, C., Treimer, W., and Mampe, W., "Single-and double-slit diffraction of neutrons," Reviews of modern physics 60(4), 1067 (1988).

[4] Taylor, G. I., "Interference fringes with feeble light," in [Proceedings of the Cambridge Philosophical Society], 15(1), 114-115 (1909).

[5] Gerlich, S., Eibenberger, S., Tomandl, M., Nimmrichter, S., Hornberger, K., Fagan, P. J., Tüxen, J., Mayor, M., and Arndt, M., "Quantum interference of large organic molecules," Nature communications 2, 263 (2011). 
[6] Bohr, N. et al., [The quantum postulate and the recent development of atomic theory], vol. 3, Printed in Great Britain by R. \& R. Clarke, Limited (1928).

[7] Wootters, W. K. and Zurek, W. H., "Complementarity in the double-slit experiment: Quantum nonseparability and a quantitative statement of bohr's principle," Physical Review D 19(2), 473 (1979).

[8] Greenberger, D. M. and Yasin, A., "Simultaneous wave and particle knowledge in a neutron interferometer," Physics Letters A 128(8), 391-394 (1988).

[9] Jaeger, G., Shimony, A., and Vaidman, L., "Two interferometric complementarities," Physical Review A 51(1), 54 (1995).

[10] Englert, B.-G., "Fringe visibility and which-way information: An inequality," Physical review letters 77(11), 2154 (1996).

[11] Scully, M. O. and Drühl, K., "Quantum eraser: A proposed photon correlation experiment concerning observation and" delayed choice" in quantum mechanics," Physical Review A 25(4), 2208 (1982).

[12] Scully, M. O., Englert, B.-G., and Walther, H., "Quantum optical tests of complementarity," Nature 351, 111-116 (1991).

[13] Walborn, S., Cunha, M. T., Pádua, S., and Monken, C., "Double-slit quantum eraser," Physical Review A 65(3), 033818 (2002).

[14] Neves, L., Lima, G., Aguirre, J., Torres-Ruiz, F., Saavedra, C., and Delgado, A., "Control of quantum interference in the quantum eraser," New Journal of Physics 11(7), 073035 (2009).

[15] Neves, L., Lima, G., Delgado, A., and Saavedra, C., "Hybrid photonic entanglement: Realization, characterization, and applications," Physical Review A 80(4), 042322 (2009).

[16] Herzog, T. J., Kwiat, P. G., Weinfurter, H., and Zeilinger, A., "Complementarity and the quantum eraser," Physical Review Letters 75(17), 3034 (1995).

[17] Kim, Y.-H., Yu, R., Kulik, S. P., Shih, Y., and Scully, M. O., "Delayed choice quantum eraser," Physical Review Letters 84(1), 1 (2000).

[18] Ma, X.-S., Kofler, J., Qarry, A., Tetik, N., Scheidl, T., Ursin, R., Ramelow, S., Herbst, T., Ratschbacher, L., Fedrizzi, A., et al., "Quantum erasure with causally disconnected choice," Proceedings of the National Academy of Sciences 110(4), 1221-1226 (2013).

[19] Ma, X.-s., Kofler, J., and Zeilinger, A., "Delayed-choice gedanken experiments and their realizations," Reviews of Modern Physics 88(1), 015005 (2016).

[20] Jacques, V., Wu, E., Grosshans, F., Treussart, F., Grangier, P., Aspect, A., and Roch, J.-F., "Delayedchoice test of quantum complementarity with interfering single photons," Physical Review Letters 100(22), 220402 (2008).

[21] Allen, L., Beijersbergen, M. W., Spreeuw, R., and Woerdman, J., "Orbital angular momentum of light and the transformation of laguerre-gaussian laser modes," Physical Review A 45(11), 8185 (1992).

[22] Allen, L., Padgett, M., and Babiker, M., "Iv the orbital angular momentum of light," Progress in optics 39, 291-372 (1999).

[23] Franke-Arnold, S., Allen, L., and Padgett, M., "Advances in optical angular momentum," Laser \&3 Photonics Reviews 2(4), 299-313 (2008).

[24] Molina-Terriza, G., Torres, J. P., and Torner, L., "Twisted photons," Nature Physics 3(5), 305-310 (2007).

[25] Nagali, E. and Sciarrino, F., "Generation of hybrid polarization-orbital angular momentum entangled states," Optics express 18(17), 18243-18248 (2010).

[26] Karimi, E., Leach, J., Slussarenko, S., Piccirillo, B., Marrucci, L., Chen, L., She, W., Franke-Arnold, S., Padgett, M. J., and Santamato, E., "Spin-orbit hybrid entanglement of photons and quantum contextuality," Physical Review A 82(2), 022115 (2010).

[27] Marrucci, L., Manzo, C., and Paparo, D., "Optical spin-to-orbital angular momentum conversion in inhomogeneous anisotropic media," Physical review letters 96(16), 163905 (2006).

[28] Marrucci, L., Karimi, E., Slussarenko, S., Piccirillo, B., Santamato, E., Nagali, E., and Sciarrino, F., "Spinto-orbital conversion of the angular momentum of light and its classical and quantum applications," Journal of Optics 13(6), 064001 (2011).

[29] Nape, I., Ndagano, B., and Forbes, A., "Erasing the orbital angular momentum information of a photon," Physical Review A 95(5), 053859 (2017). 
[30] Leach, J., Jack, B., Romero, J., Ritsch-Marte, M., Boyd, R., Jha, A., Barnett, S., Franke-Arnold, S., and Padgett, M., "Violation of a bell inequality in two-dimensional orbital angular momentum state-spaces," Optics express 17(10), 8287-8293 (2009).

[31] Dada, A. C., Leach, J., Buller, G. S., Padgett, M. J., and Andersson, E., "Experimental high-dimensional two-photon entanglement and violations of generalized bell inequalities," Nature Physics 7(9), 677-680 (2011).

[32] Agnew, M., Leach, J., McLaren, M., Roux, F. S., and Boyd, R. W., "Tomography of the quantum state of photons entangled in high dimensions," Physical Review A 84(6), 062101 (2011).

[33] Fickler, R., Lapkiewicz, R., Plick, W. N., Krenn, M., Schaeff, C., Ramelow, S., and Zeilinger, A., "Quantum entanglement of high angular momenta," Science 338(6107), 640-643 (2012).

[34] Forbes, A., Dudley, A., and McLaren, M., "Creation and detection of optical modes with spatial light modulators," Advances in Optics and Photonics 8(2), 200-227 (2016).

[35] Rubinsztein-Dunlop, H., Forbes, A., Berry, M., Dennis, M., Andrews, D. L., Mansuripur, M., Denz, C., Alpmann, C., Banzer, P., Bauer, T., et al., "Roadmap on structured light," Journal of Optics 19(1), 013001 (2016).

[36] Nape, I., Kyeremah, C., Vallés, A., Rosales-Guzmán, C., Buah-Bassuah, P. K., and Forbes, A., "A hybrid quantum eraser scheme for characterization of free-space and fiber communication channels," Optics Communications (2017).

[37] Ndagano, B., Perez-Garcia, B., Roux, F. S., McLaren, M., Rosales-Guzman, C., Zhang, Y., Mouane, O., Hernandez-Aranda, R. I., Konrad, T., and Forbes, A., "Characterizing quantum channels with non-separable states of classical light," Nature Physics 13(4), 397-402 (2017).

[38] Ndagano, B., Brüning, R., McLaren, M., Duparré, M., and Forbes, A., "Fiber propagation of vector modes," Optics express 23(13), 17330-17336 (2015).

[39] Nape, I., Ndagano, B., Perez-Garcia, B., Scholes, S., Hernandez-Aranda, R. I., Konrad, T., and Forbes, A., "High-bit-rate quantum key distribution with entangled internal degrees of freedom of photons," arXiv preprint arXiv:1612.09261 (2016). 